\title{
ON SEMISTABLE PRINCIPAL BUNDLES OVER A COMPLEX PROJECTIVE MANIFOLD
}

\author{
INDRANIL BISWAS AND UGO BRUZZO
}

\begin{abstract}
Let $G$ be a simple linear algebraic group defined over the field of complex numbers. Fix a proper parabolic subgroup $P$ of $G$, and also fix a nontrivial antidominant character $\chi$ of $P$. We prove that a holomorphic principal $G$-bundle $E_{G}$ over a connected complex projective manifold $M$ is semistable satisfying the condition that the second Chern class $c_{2}\left(\operatorname{ad}\left(E_{G}\right)\right) \in H^{4}(M, \mathbb{Q})$ vanishes if and only if the line bundle over $E_{G} / P$ defined by $\chi$ is numerically effective. Also, a principal $G$-bundle $E_{G}$ over $M$ is semistable with $c_{2}\left(\operatorname{ad}\left(E_{G}\right)\right)=0$ if and only if for every pair of the form $(Y, \psi)$, where $\psi$ is a holomorphic map to $M$ from a compact connected Riemann surface $Y$, and for every holomorphic reduction of structure group $E_{P} \subset \psi^{*} E_{G}$ to the subgroup $P$, the line bundle over $Y$ associated to the principal $P$-bundle $E_{P}$ for $\chi$ is of nonnegative degree. Therefore, $E_{G}$ is semistable with $c_{2}\left(\operatorname{ad}\left(E_{G}\right)\right)=0$ if and only if for each pair $(Y, \psi)$ of the above type the $G$-bundle $\psi^{*} E_{G}$ over $Y$ is semistable.

Similar results remain valid for principal bundles over $M$ with a reductive linear algebraic group as the structure group. These generalize an earlier work of Y. Miyaoka, [Mi], where he gave a characterization of semistable vector bundles over a smooth projective curve. Using these characterizations one can also produce similar criteria for the semistability of parabolic principal bundles over a compact Riemann surface.
\end{abstract}

\section{INTRODUCTION}

Let $G$ be a simple linear algebraic group defined over $\mathbb{C}$. Fix a proper parabolic subgroup $P$ of $G$. Fix a nontrivial antidominant character $\chi$ of $P$. This means that the associated line bundle $\left(G \times \mathbb{C}_{\chi}\right) / P$ over $G / P$ is numerically effective and nontrivial.

Let $M$ be a connected smooth complex projective variety. Let $E_{G}$ be a holomorphic principal $G$-bundle over $M$. The natural projection $E_{G} \longrightarrow E_{G} / P$ defines a principal $P$-bundle over $E_{G} / P$, and hence the character $\chi$ of $P$ associates a holomorphic line bundle

$$
L_{\chi}=\left(E_{G} \times \mathbb{C}\right) / P
$$

over $E_{G} / P$, where the action of $p \in P$ sends any $(z, c) \in E_{G} \times \mathbb{C}$ to $\left(z p, \chi(p)^{-1} c\right)$.

We prove the following criterion for $L_{\chi}$ to be numerically effective (see Theorem 3.2):

Theorem 1.1. The line bundle $L_{\chi}$ over $E_{G} / P$ is numerically effective if and only if $E_{G}$ is semistable and $c_{2}\left(a d\left(E_{G}\right)\right)=0$.

It should be clarified that this criterion for semistability of any $G$-bundle $E_{G}$ with $c_{2}\left(\operatorname{ad}\left(E_{G}\right)\right)=0$ needs to be verified for just one pair $(P, \chi)$. Since the condition

2000 Mathematics Subject Classification. 32L05, 14L10, 14F05.

Key words and phrases. Principal bundle, semistability, numerically effectiveness. 
$c_{2}\left(\operatorname{ad}\left(E_{G}\right)\right)=0$ is automatically satisfied when $M$ is a Riemann surface, Theorem 3.2 gives a characterization of semistable $G$-bundles over a compact connected Riemann surface.

In the special case where $\operatorname{dim} M=1, G=\mathrm{GL}(n, \mathbb{C})$, and $P$ is the parabolic subgroup of $\operatorname{GL}(n, \mathbb{C})$ that fixes a line in $\mathbb{C}^{n}$ (so $G / P=\mathbb{C P}^{n-1}$ ), Theorem 3.2 is due to Y. Miyaoka (see [Mi, page 456, Theorem 3.1]); in this case $\operatorname{Pic}(G / P)=\mathbb{Z}$ and hence $\chi$ is unique up to taking tensor powers. In $[\mathrm{BH}]$, Miyaoka's result was generalized to the context of Higgs vector bundles over curves.

As a consequence of Theorem 3.2, the line bundle $L_{\chi}$ over $E_{G} / P$ is numerically effective if and only if for every parabolic subgroup $P^{\prime} \subset G$, and every nontrivial antidominant character $\chi^{\prime}$ of $P^{\prime}$, the associated line bundle $L_{\chi^{\prime}}:=\left(E_{G} \times \mathbb{C}_{\chi^{\prime}}\right) / P^{\prime}$ over $E_{G} / P^{\prime}$ is numerically effective. The above condition that $c_{2}\left(\operatorname{ad}\left(E_{G}\right)\right) \in H^{4}(M, \mathbb{Q})$ vanishes is equivalent to the condition that the real characteristic class of $E_{G}$ corresponding to the Killing form on the Lie algebra of $G$ vanishes (Remark 3.3).

In Section 2 we prove Theorem 3.2 under the assumption that $\operatorname{dim} M=1$ (see Proposition 2.2). The proof of Theorem 3.2 given in Section 3 crucially uses this special case.

Theorem 3.2 can be reformulated as follows:

A $G$-bundle $E_{G}$ over $M$ is semistable with $c_{2}\left(\operatorname{ad}\left(E_{G}\right)\right)=0$ if and only if for every pair of the form $(Y, \psi)$, where $Y$ is a compact connected Riemann surface and

$$
\psi: Y \longrightarrow M
$$

a holomorphic map, and for every reduction $E_{P} \subset \psi^{*} E_{G}$ of structure group, to the fixed parabolic subgroup $P$, of the principal $G$-bundle $\psi^{*} E_{G}$ over $Y$, the associated line bundle $E_{P}(\chi)=\left(E_{P} \times \mathbb{C}\right) / P$ over $Y$ is of nonnegative degree, where $\chi$ is the fixed character of $P$ (Proposition 4.1).

It is known that a $G$-bundle $E_{G}$ over $M$ is semistable if and only if the restriction of $E_{G}$ to the general complete intersection curve of sufficiently large degree hypersurfaces is semistable. Therefore, we may ask the following question: under what condition the restriction of $E_{G}$ to every smooth curve in $M$ is semistable? As a consequence of Proposition 4.1 and Theorem 3.2 this question has the following answer:

$A G$-bundle $E_{G}$ is semistable and $c_{2}\left(\operatorname{ad}\left(E_{G}\right)\right)=0$ if and only if for each pair $(Y, \psi)$ of the above type the $G$-bundle $\psi^{*} E_{G}$ over $Y$ is semistable.

In Section 4 we give the following analog of Theorem 3.2 and Proposition 4.1 for principal bundles with a reductive group as the structure group (see Theorem 4.3):

Theorem 1.2. Let $G$ be a connected reductive linear algebraic group over $\mathbb{C}$. Fix a parabolic subgroup $P \subset G$ without any simple factor, and also fix a character $\chi$ of $P$ such that

(i) $\chi$ is trivial on the center $Z(G) \subset G$, and

(ii) the restriction of $\chi$ to the parabolic subgroup of each simple factor of $G / Z(G)$ defined by $P$ is nontrivial and antidominant. 
Let $E_{G}$ be a principal $G$-bundle over a connected projective manifold $M$. Then the following four statements are equivalent:

(1) The $G$-bundle $E_{G}$ is semistable and the second Chern class

$$
c_{2}\left(\operatorname{ad}\left(E_{G}\right)\right) \in H^{4}(M, \mathbb{Q})
$$

vanishes.

(2) The associated line bundle $L_{\chi}:=\left(E_{G} \times \mathbb{C}_{\chi}\right) / P$ over $E_{G} / P$ for the character $\chi$ is numerically effective.

(3) For every pair of the form $(Y, \psi)$, where $Y$ is a compact connected Riemann surface and

$$
\psi: Y \longrightarrow M
$$

a holomorphic map, and every reduction $E_{P} \subset \psi^{*} E_{G}$ of structure group to $P$ of the principal $G$-bundle $\psi^{*} E_{G}$ over $Y$, the associated line bundle $E_{P}(\chi)=\left(E_{P} \times\right.$ $\left.\mathbb{C}_{\chi}\right) / P$ over $Y$ is of nonnegative degree.

(4) For any pair $(Y, \psi)$ as in (3), the $G$-bundle $\psi^{*} E_{G}$ over $Y$ is semistable.

Let $G$ be a connected reductive linear algebraic group over $\mathbb{C}$. A parabolic G-bundle $E_{*}$ over a connected smooth complex projective curve $X$ with parabolic structure over a reduced divisor $D \subset X$ is, loosely speaking, a smooth quasiprojective variety $E_{G}^{\prime}$ carrying an action of $G$ together with a dominant morphism $\psi: E_{G}^{\prime} \longrightarrow X$ such that $E_{G}^{\prime}$ is a principal $G$-bundle over $X \backslash D$, and the isotropy groups corresponding to the action of $G$ on the fibers of $\psi$ over the points of $D$ are finite (for a precise definition see Section 5). Let $P$ be a proper parabolic subgroup of $G$ and $\chi: P \longrightarrow \mathbb{G}_{m}=\mathbb{C}^{*}$ a nontrivial antidominant character. Denote by $N$ the least common multiple of the orders of the isotropy groups for the action of $G$ on $E_{G}^{\prime}$. Then the character $\chi^{N}$ defines a line bundle over $E_{G}^{\prime} / P$. We show that the parabolic bundle is semistable if and only if this line bundle over $E_{G}^{\prime} / P$ is numerically effective (Proposition 5.1).

\section{Criterion For Semistability over a CURVE}

Let $G$ be a simple linear algebraic group defined over the field of complex numbers. A parabolic subgroup of $G$ is a connected Zariski closed proper subgroup $P \subsetneq G$ such that $G / P$ is a complete variety. The quotient map $G \longrightarrow G / P$ defines a holomorphic principal $P$-bundle over $G / P$. We recall that a character $\chi$ of $P$ is called antidominant if the line bundle $\left(G \times \mathbb{C}_{\chi}\right) / P$ over $G / P$, associated to this $P$-bundle for the character $\chi$, is numerically effective; here the action of any $p \in P$ sends $(g, \lambda) \in G \times \mathbb{C}$ to $\left(g p, \chi(p)^{-1} \lambda\right)$. Note that the character group of $P$ is identified with a finite index subgroup of $\operatorname{Pic}(G / P)$.

Let $X$ be a connected smooth projective curve defined over $\mathbb{C}$. We recall from [Ra, page 129 , Definition 1.1] that a holomorphic $G$-bundle $E_{G}$ over the curve $X$ is called semistable if for every reduction of structure group of $E_{G}$

$$
\sigma: X \longrightarrow E_{G} / P
$$

to a maximal parabolic subgroup $P$ of $G$ one has

$$
\operatorname{degree}\left(\sigma^{*} T_{\text {rel }}\right) \geq 0,
$$


where $T_{\text {rel }}$ is the relative tangent bundle over $E_{G} / P$ for the natural projection $E_{G} / P \longrightarrow$ $X$. Alternatively, one can say that $E_{G}$ is semistable if for every triple $\left(P, E_{P}, \chi\right)$, where $P \subset G$ is a parabolic subgroup, $E_{P} \subset E_{G}$ a reduction of structure group of $E_{G}$ to $P$ and $\chi$ an antidominant character of $P$, the associated line bundle $E_{P}(\chi)=\left(E_{P} \times \mathbb{C}\right) / P$ over $X$ is of nonnegative degree [Ra, page 131, Lemma 2.1].

The following lemma will be needed in the proof of Proposition 2.2 .

Lemma 2.1. A principal $G$-bundle $E_{G}$ over $X$ is semistable if and only if there is a nontrivial finite dimensional complex $G$-module $V$ such that the vector bundle $E_{G}(V):=$ $\left(E_{G} \times V\right) / G$ over $X$ associated to $E_{G}$ for $V$ is semistable.

Proof. If $E_{G}$ is semistable, from $[\mathrm{RR}$, page 285, Theorem 3.18] it follows immediately that the associated vector bundle $E_{G}(V):=\left(E_{G} \times V\right) / V$ is semistable.

Now assume that $E_{G}(V)$ is semistable, where $V$ is a nontrivial finite dimensional complex $G$-module.

Take a maximal parabolic subgroup $Q \subset G$. There is a parabolic subgroup $P_{1} \subset$ $\operatorname{SL}(V)$ such that $Q=P_{1} \bigcap G$ (so $G / Q$ is embedded in $\operatorname{SL}(V) / P_{1}$ ), and some positive multiple of any given ample line bundle over $G / Q$ is the restriction of some ample line bundle on $\operatorname{SL}(V) / P_{1}$. To prove this assertion, consider the Jordan-Hölder filtration of $V$ for the action of the unipotent radical of $Q$ on $V$ (the unipotent radical acts trivially on each successive quotient of the Jordan-Hölder filtration); since the unipotent radical is a normal subgroup of $Q$, the action of $Q$ on $V$ preserves this filtration. The above parabolic subgroup $P_{1}$ can be taken to be the subgroup of $\operatorname{SL}(V)$ that preserves this filtration of $V$. Note that since $\operatorname{Pic}(G / Q) \cong \mathbb{Z}$, the restriction to $G / Q$ of any ample line bundle over $\mathrm{SL}(V) / P_{1}$ is a positive multiple of the (unique) ample generator of $\operatorname{Pic}(G / Q)$.

Therefore, if $\sigma: X \longrightarrow E_{G} / Q$ is a reduction of structure group of $E_{G}$ to $Q$, then degree $\left(\sigma^{*} T_{G / Q}\right)$ is a positive multiple of $\operatorname{degree}\left(\sigma_{1}^{*} T_{\mathrm{SL}(V) / P_{1}}\right)$, where

$$
\sigma_{1}: X \longrightarrow E_{G}(\mathrm{SL}(V)) / P_{1}
$$

is the reduction of structure group to $P_{1} \subset \mathrm{SL}(V)$, defined by $\sigma$, of the principal $\mathrm{SL}(V)-$ bundle obtained by extending the structure group of $E_{G}$ (by the injective homomorphism $V \longrightarrow \mathrm{SL}(V)$ ), and $T_{G / Q}$ (respectively, $T_{\mathrm{SL}(V) / P_{1}}$ ) is the relative tangent bundle over $E_{G} / Q$ (respectively, $\left.E_{G}(\mathrm{SL}(V)) / P_{1}\right)$ for the natural projection to $X$. Consequently, the $G$-bundle $E_{G}$ is semistable if the vector bundle $E_{G}(V)$ is so. This completes the proof of the lemma.

For a principal $G$-bundle $E_{G}$ over $X$ the quotient $E_{G} / P$ is a fiber bundle over $X$ with fiber $G / P$, and furthermore, the projection $E_{G} \longrightarrow E_{G} / P$ defines a principal $P$-bundle over $E_{G} / P$. For any character $\chi$ of $P$, let

$$
L_{\chi}:=\left(E_{G} \times \mathbb{C}_{\chi}\right) / P
$$

be the line bundle over $E_{G} / P$ associated to this $P$-bundle for the character $\chi$ of $P$; the action of $P$ on $E_{G} \times \mathbb{C}_{\chi}$ is defined as before. 
Proposition 2.2. A principal $G$-bundle $E_{G}$ over $X$ is semistable if and only if there is a pair $(P, \chi)$, where $P \subset G$ is a proper parabolic subgroup and

$$
\chi: P \longrightarrow \mathbb{G}_{m}=\mathbb{C}^{*}
$$

a nontrivial antidominant character, such that the associated line bundle $L_{\chi}$ over $E_{G} / P$ (see Eq. (2.1)) is numerically effective.

Proof. First assume that $E_{G}$ is semistable.

Let

$$
f: E_{G} / P \longrightarrow X
$$

be the natural projection. Any fiber of $f$ is isomorphic to $G / P$, and the restriction of $L_{\chi}$, defined in Eq. (2.1), to a fiber of $f$ is isomorphic to the associated line bundle $(G \times \mathbb{C}) / P$ over $G / P$ corresponding to $\chi$. As $\chi$ is antidominant, we conclude that the restriction of $L_{\chi}$ to a fiber of $f$ is numerically effective.

Let $Y_{0} \subset E_{G} / P$ be an irreducible curve which is not contained in any fiber of $f$. Let $\iota: Y \longrightarrow Y_{0}$ be the normalization. Therefore, $Y$ is a smooth curve, and the map

$$
f_{Y}:=f \circ \iota: Y \longrightarrow X
$$

makes $Y$ a (possibly ramified) covering of $X$. Note that there is a tautological section

$$
\sigma: Y \longrightarrow\left(f_{Y}^{*} E_{G}\right) / P \cong f_{Y}^{*}\left(E_{G} / P\right)
$$

that sends any $y \in Y$ to the point of $f_{Y}^{*}\left(E_{G} / P\right)$ defined by $\iota(y) \in E_{G} / P$. Let $E_{P}^{Y} \subset f_{Y}^{*} E_{G}$ be the reduction of structure group to $P$, of the $G$-bundle $f_{Y}^{*} E_{G}$ over $Y$, defined by $\sigma$.

Note that the $P$-bundle $E_{P}^{Y}$ is identified with the principal $P$-bundle $\iota^{*} E_{G}$, namely the pullback to $Y$ of the principal $P$-bundle $E_{G} \longrightarrow E_{G} / P$. Therefore, the pullback $\iota^{*} L_{\chi}$ over $Y$ is naturally identified with the line bundle $E_{P}^{Y}(\chi)$ associated to $E_{P}^{Y}$ for the character $\chi$ of $P$. Consequently, to prove that $L_{\chi}$ is numerically effective it is enough to show that the $G$-bundle $f_{Y}^{*} E_{G}$ is semistable, where $f_{Y}$ is defined in Eq. (2.3).

Any finite index subgroup of a finitely presented group $\Gamma$ contains a normal subgroup of $\Gamma$ of finite index. Therefore we have a (possibly ramified) covering $Y^{\prime} \longrightarrow Y$ such that the composition

$$
Y^{\prime} \longrightarrow Y \longrightarrow X
$$

which we will denote by $f_{1}$, is a finite Galois covering. Let $\Gamma_{0}=\operatorname{Gal}\left(Y^{\prime} / X\right)$ denote the Galois group for $f_{1}$. It is easy to see that if $f_{1}^{*} E_{G}$ is semistable, then $f_{Y}^{*} E_{G}$ is semistable (the pull back to $Y^{\prime}$ of a destabilizing reduction of $f_{Y}^{*} E_{G}$ destabilizes $f_{1}^{*} E_{G}$ ).

If $f_{1}^{*} E_{G}$ is not semistable, then it admits a Harder-Narasimhan reduction of structure group $E_{Q}^{\prime} \subset f_{1}^{*} E_{G}$ to a proper parabolic subgroup $Q \subset G$ AAB, page 694, Theorem 1]. The uniqueness of the Harder-Narasimhan reduction implies that the natural action of the Galois group $\Gamma_{0}$ on the total space of $f_{1}^{*} E_{G}$ (lifting the action of $\Gamma_{0}$ on $Y^{\prime}$ ) leaves the submanifold $E_{Q}^{\prime}$ invariant. Consequently, the reduction $E_{Q}^{\prime} \subset f_{1}^{*} E_{G}$ descends to a reduction of structure group of $E_{G}$ to $Q$. Since this reduction of structure group to $Q$ satisfies all the conditions of a Harder-Narasimhan reduction, the $G$-bundle $E_{G}$ is not semistable. In other words, we have a contradiction, and hence $f_{1}^{*} E_{G}$ is semistable. 
Therefore, the $G$-bundle $f_{Y}^{*} E_{G}$ over $Y$ is semistable. As we saw earlier, this implies that $L_{\chi}$ over $E_{G} / P$ is numerically effective.

To prove the converse, let $P$ and $\chi$ be such that the line bundle $L_{\chi}$ over $E_{G} / P$ is numerically effective and nontrivial. Let $\theta_{\chi}:=\left(G \times \mathbb{C}_{\chi}\right) / P$ be the associated line bundle over $G / P$, defined by $\chi$, associated to the $P$-bundle given by the natural projection $G \longrightarrow G / P$. Since $\chi$ is antidominant, the line bundle $\theta_{\chi}$ is numerically effective, and $\theta_{\chi}$ is nontrivial as $\chi$ is so.

Let

$$
V:=H^{0}\left(G / P, \theta_{\chi}\right)
$$

be the irreducible $G$-module (the irreducibility is a part of the Borel-Weil theorem; see [PS, page 21, Theorem 2.9.1(ii)]). Note that since $\theta_{\chi}$ is nontrivial, the $G$-module $V$ is also nontrivial. We also know that for any $n \geq 1$, the irreducible $G$-module

$$
V_{n}:=H^{0}\left(G / P, \theta_{\chi}^{\otimes n}\right)
$$

is a direct summand of the $G$-module $V^{\otimes n}$. To see this note that there is a set-theoretic map from $V$ to $V_{n}$ that sends any section

$$
s \in H^{0}\left(G / P, \theta_{\chi}\right)
$$

to $s^{\otimes n} \in H^{0}\left(G / P, \theta_{\chi}^{\otimes n}\right)$. The linear span of the image of this map is a quotient of the symmetric product $\operatorname{Sym}^{n}\left(H^{0}\left(G / P, \theta_{\chi}\right)\right)$. Therefore, the $G$-module $V_{n}$ is a direct summand of $\operatorname{Sym}^{n}(V)$. Since the $G$-module $\operatorname{Sym}^{n}(V)$ is a direct summand of $V^{\otimes n}$, we conclude that $V_{n}$ is a direct summand of $V^{\otimes n}$.

Let $E_{G}(V):=\left(E_{G} \times V\right) / G$ be the vector bundle over $X$ associated to $E_{G}$ for the $G$-module $V$. In view of Lemma 2.1, to prove that $E_{G}$ is semistable it suffices to show that the vector bundle $E_{G}(V)$ is semistable.

The vector bundle $E_{G}(V)$ is clearly identified with the direct image $f_{*} L_{\chi}$, and similarly $E_{G}\left(V_{n}\right)$ is identified with $f_{*} L_{\chi}^{\otimes n}$, where $V$ and $V_{n}$ are defined in Eq. (2.4) and Eq. (2.5) respectively, and $f$ is the projection in Eq. (2.2). The group $G$ being simple does not have any nontrivial character. Hence the degree of every vector bundle associated to $E_{G}$ is zero.

Assume that the vector bundle $E_{G}(V) \cong f_{*} L_{\chi}$ is not semistable. Let $F \subset f_{*} L_{\chi}$ be the (unique) maximal semistable subbundle $E_{G}(V)$. So $F$ is the first term of the HarderNarasimhan filtration of $E_{G}(V)$. Since $\operatorname{degree}\left(E_{G}(V)\right)=0$, we have degree $(F)>0$. For any $n \geq 1$, let

$$
F_{n} \subset f_{*} L_{\chi}^{\otimes n} \cong E_{G}\left(V_{n}\right)
$$

be the coherent subsheaf generated by $\left\{v^{\otimes n}\right\}_{v \in F}$. This subsheaf $F_{n}$ is a quotient of the symmetric product $\operatorname{Sym}^{n}(F)$. Note that

$$
\frac{\operatorname{degree}\left(\operatorname{Sym}^{n}(F)\right)}{\operatorname{rank}\left(\operatorname{Sym}^{n}(F)\right)}=\frac{n \cdot \operatorname{degree}(F)}{\operatorname{rank}(F)},
$$


and since $F$ is semistable, the symmetric product $\operatorname{Sym}^{n}(F)$ is semistable [RR, page 285, Theorem 3.18]. Consequently, for the quotient $F_{n}$ of $\operatorname{Sym}^{n}(F)$ we have

$$
\frac{\operatorname{degree}\left(F_{n}\right)}{\operatorname{rank}\left(F_{n}\right)} \geq \frac{n \cdot \operatorname{degree}(F)}{\operatorname{rank}(F)} \text {. }
$$

Let $p_{0} / q_{0}$, with $p_{0}, q_{0} \in \mathbb{N}$, be a positive rational number such that

$$
\frac{p_{0}}{q_{0}}<\frac{\operatorname{degree}(F)}{\operatorname{rank}(F)}
$$

(recall that $\frac{\operatorname{degree}(F)}{\operatorname{rank}(F)}>0$ ). Fix a point $x_{0} \in X$. For any integer $m \geq 1$, consider the vector bundle

$$
W_{m}:=F_{m q_{0}} \otimes \mathcal{O}_{X}\left(-m p_{0} x_{0}\right)
$$

over $X$ where $F_{m q_{0}}$ is defined in Eq. (2.6). Using Eq. (2.7) we have

$$
\frac{\operatorname{degree}\left(W_{m}\right)}{\operatorname{rank}\left(W_{m}\right)} \geq m q_{0}\left(\frac{\operatorname{degree}(F)}{\operatorname{rank}(F)}-\frac{p_{0}}{q_{0}}\right)>0 \text {. }
$$

In view of this inequality, if $m$ is such that $m q_{0}\left(\frac{\operatorname{degree}(F)}{\operatorname{rank}(F)}-\frac{p_{0}}{q_{0}}\right)>\operatorname{genus}(X)-1$, then the Riemann-Roch theorem gives

$$
\operatorname{dim} H^{0}\left(X, W_{m}\right) \geq \chi\left(W_{m}\right) \geq \operatorname{rank}\left(W_{m}\right)\left(m q_{0}\left(\frac{\operatorname{degree}(F)}{\operatorname{rank}(F)}-\frac{p_{0}}{q_{0}}\right)-\operatorname{genus}(X)+1\right)>0 .
$$

Take an integer $m$ such that $m q_{0}\left(\frac{\operatorname{degree}(F)}{\operatorname{rank}(F)}-\frac{p_{0}}{q_{0}}\right)>\operatorname{genus}(X)-1$, and take a nonzero section

$$
0 \neq s_{0} \in H^{0}\left(X, W_{m}\right) \subset H^{0}\left(X, E_{G}\left(V_{m q_{0}}\right) \otimes \mathcal{O}_{X}\left(-m p_{0} x_{0}\right)\right) .
$$

Using the isomorphism $f_{*} L_{\chi}^{\otimes n} \cong E_{G}\left(V_{n}\right)$ and the projection formula we have

$$
H^{0}\left(X, E_{G}\left(V_{m q_{0}}\right) \otimes \mathcal{O}_{X}\left(-m p_{0} x_{0}\right)\right)=H^{0}\left(E_{G} / P, L_{\chi}^{\otimes m q_{0}} \otimes f^{*} \mathcal{O}_{X}\left(-m p_{0} x_{0}\right)\right) .
$$

Let

$$
s_{0}^{\prime} \in H^{0}\left(E_{G} / P, L_{\chi}^{\otimes m q_{0}} \otimes f^{*} \mathcal{O}_{X}\left(-m p_{0} x_{0}\right)\right)
$$

be the section defined by $s_{0}$ (in Eq. (2.8)) using the above isomorphism. Set

$$
\operatorname{Div}\left(s_{0}^{\prime}\right) \subset E_{G} / P
$$

to the effective divisor defined by the above section $s_{0}^{\prime}$.

Let $d_{0}$ be the largest integer such that the cup product $c_{1}\left(\theta_{\chi}\right)^{d_{0}} \in H^{2 d_{0}}(G / P$, $\mathbb{Q})$ is nonzero. Since $\theta_{\chi}$ is numerically effective but nontrivial, there is a parabolic subgroup $P^{\prime} \supseteq P$ of $G$ such that $\theta_{\chi}$ is the pull back of an ample line bundle over $G / P^{\prime}$ using the natural projection of $G / P$ to $G / P^{\prime}$. The above defined integer $d_{0}$ is the dimension of $G / P^{\prime}$.

Let $\chi^{\prime}$ be the character of $P^{\prime}$ that restricts to $\chi$ on $P$. Equivalently, the line bundle $\theta_{\chi^{\prime}}$ over $G / P^{\prime}$ defined by $\chi^{\prime}$ satisfies the condition that $\theta_{\chi^{\prime}}$ pulls back to $\theta_{\chi}$.

Let

$$
\phi: E_{G} / P \longrightarrow E_{G} / P^{\prime}
$$


be the natural projection and $L_{\chi^{\prime}}:=\left(E_{G} \times \mathbb{C}_{\chi^{\prime}}\right) / P^{\prime}$ the line bundle over $E_{G} / P^{\prime}$ defined by $\chi^{\prime}$. So, we have $\phi^{*} L_{\chi^{\prime}} \cong L_{\chi}$.

Since $L_{\chi}$ is numerically effective and $\phi$ is surjective, we conclude that $L_{\chi^{\prime}}$ is numerically effective [Fu, page 360, Proposition 2.3].

Since $\phi_{*} \mathcal{O}_{E_{G} / P} \cong \mathcal{O}_{E_{G} / P^{\prime}}$, we have

$$
\phi_{*}\left(L_{\chi}^{\otimes m q_{0}} \otimes f^{*} \mathcal{O}_{X}\left(-m p_{0} x_{0}\right)\right) \cong L_{\chi^{\prime}}^{\otimes m q_{0}} \otimes h^{*} \mathcal{O}_{X}\left(-m p_{0} x_{0}\right),
$$

where

$$
h: E_{G} / P^{\prime} \longrightarrow X
$$

is the natural projection. Therefore,

$$
H^{0}\left(E_{G} / P, L_{\chi}^{\otimes m q_{0}} \otimes f^{*} \mathcal{O}_{X}\left(-m p_{0} x_{0}\right)\right) \cong H^{0}\left(E_{G} / P^{\prime}, L_{\chi^{\prime}}^{\otimes m q_{0}} \otimes h^{*} \mathcal{O}_{X}\left(-m p_{0} x_{0}\right)\right) .
$$

Consequently, the section $s_{0}^{\prime}$ of $L_{\chi}^{\otimes m q_{0}} \otimes f^{*} \mathcal{O}_{X}\left(-m p_{0} x_{0}\right)$ constructed in Eq. (2.9) corresponds to a section

$$
\zeta_{0} \in H^{0}\left(E_{G} / P^{\prime}, L_{\chi^{\prime}}^{\otimes m q_{0}} \otimes h^{*} \mathcal{O}_{X}\left(-m p_{0} x_{0}\right)\right),
$$

and the divisor $\operatorname{Div}\left(s_{0}^{\prime}\right)$ on $E_{G} / P$ coincides with the divisor $\phi^{-1}\left(\operatorname{Div}\left(\zeta_{0}\right)\right)$.

We will show that $\left(c_{1}\left(L_{\chi^{\prime}}\right)\right)^{d_{0}+1} \in H^{2 d_{0}+2}\left(E_{G} / P^{\prime}, \mathbb{Z}\right) \cong \mathbb{Z}$ vanishes.

The topological isomorphism classes of $G$-bundles over a compact connected Riemann surface are parametrized by the fundamental group $\pi_{1}(G)$ [Ra, page 142, Proposition 5.1]. Since $G$ is simple, we know that $\pi_{1}(G)$ is a finite group. Therefore, if $\beta: X^{\prime} \longrightarrow X$ is a finite cover (possibly ramified) of degree $\# \pi_{1}(G)$ with $X^{\prime}$ connected, then $\beta^{*} E_{G}$ is topologically trivial; here $\# \pi_{1}(G)$ is the cardinality of $\pi_{1}(G)$. Fix a covering

$$
\beta: X^{\prime} \longrightarrow X
$$

such that $\beta^{*} E_{G}$ is topologically trivial.

Let $\widehat{\beta}: \beta^{*} E_{G} / P^{\prime} \longrightarrow E_{G} / P^{\prime}$ be the natural projection over $\beta$. So $\widehat{\beta}^{*} L_{\chi^{\prime}}$ is isomorphic to the line bundle $\beta^{*} E_{G}\left(\chi^{\prime}\right):=\left(\beta^{*} E_{G} \times \mathbb{C}_{\chi^{\prime}}\right) / P^{\prime}$ (the line bundle over $\beta^{*} E_{G} / P^{\prime}$ associated to the principal $P^{\prime}$-bundle defined by $\beta^{*} E_{G} \longrightarrow \beta^{*} E_{G} / P^{\prime}$ for the character $\chi^{\prime}$ ). If we fix a topological isomorphism

$$
\tau: \beta^{*} E_{G} \longrightarrow X^{\prime} \times G
$$

of $\beta^{*} E_{G}$ with the trivial $G$-bundle over $X^{\prime}$, then the line bundle $\beta^{*} E_{G}\left(\chi^{\prime}\right)$ over $\beta^{*} E_{G} / P^{\prime}$ is identified with the pullback $\left(p_{G / P^{\prime}} \circ \tau\right)^{*} \mathcal{L}_{P^{\prime}}$, where $p_{G / P^{\prime}}$ is the composition of the projection of $X^{\prime} \times G \longrightarrow G$ with the projection $G \longrightarrow G / P$, and $\mathcal{L}_{P^{\prime}}$ is the line bundle over $G / P^{\prime}$ defined by $\chi^{\prime}$. Therefore, we have

$$
\left(c_{1}\left(\beta^{*} E_{G}\left(\chi^{\prime}\right)\right)\right)^{d_{0}+1}=0 .
$$

Since $\left(c_{1}\left(\beta^{*} E_{G}\left(\chi^{\prime}\right)\right)\right)^{d_{0}+1} \in \mathbb{Q}$ coincides with $\# \pi_{1}(G) \cdot\left(c_{1}\left(L_{\chi^{\prime}}\right)\right)^{d_{0}+1}$ (the degree of $\widehat{\beta}$ coincides with the degree of $\beta$, and the degree of $\beta$ is $\left.\# \pi_{1}(G)\right)$, this implies that $\left(c_{1}\left(L_{\chi^{\prime}}\right)\right)^{d_{0}+1}=$ 0 .

Since the cohomology class

$$
\left.c_{1}\left(L_{\chi^{\prime}}^{\otimes m q_{0}}\right)\right)^{d_{0}+1}=\left(m q_{0}\right)^{d_{0}+1}\left(c_{1}\left(L_{\chi^{\prime}}\right)\right)^{d_{0}+1} \in H^{2 d_{0}+2}\left(E_{G} / P^{\prime}, \mathbb{Z}\right)
$$


is zero, it can be shown that the integer

$$
\left(c_{1}\left(L_{\chi^{\prime}}^{\otimes m q_{0}}\right)\right)^{d_{0}} c_{1}\left(L_{\chi^{\prime}}^{\otimes m q_{0}} \otimes h^{*} \mathcal{O}_{X}\left(-m p_{0} x_{0}\right)\right) \in H^{2 d_{0}+2}\left(E_{G} / P^{\prime}, \mathbb{Z}\right) \cong \mathbb{Z}
$$

coincides with

$$
-m p_{0}\left(c_{1}\left(\theta_{\chi^{\prime}}\right)\right)^{d_{0}} \in H^{2 d_{0}}\left(G / P^{\prime}, \mathbb{Z}\right) \cong \mathbb{Z}
$$

where $d_{0}$, as before, is the dimension of $G / P^{\prime}$ and $h$ is the projection in Eq. (2.10). Indeed, we have $c_{1}\left(L_{\chi^{\prime}}^{\otimes m q_{0}} \otimes h^{*} \mathcal{O}_{X}\left(-m p_{0} x_{0}\right)\right)=c_{1}\left(L_{\chi^{\prime}}^{\otimes m q_{0}}\right)-m p_{0}\left[h^{-1}\left(x_{0}\right)\right]$, where $\left[h^{-1}\left(x_{0}\right)\right]$ is cohomology class defined by $h^{-1}\left(x_{0}\right)$ using Poincaré duality. Hence

$$
\begin{gathered}
\left(c_{1}\left(L_{\chi^{\prime}}^{\otimes m q_{0}}\right)\right)^{d_{0}} c_{1}\left(L_{\chi^{\prime}}^{\otimes m q_{0}} \otimes h^{*} \mathcal{O}_{X}\left(-m p_{0} x_{0}\right)\right) \\
\left.=c_{1}\left(L_{\chi^{\prime}}^{\otimes m q_{0}}\right)\right)^{d_{0}+1}-m p_{0}\left(c_{1}\left(\theta_{\chi^{\prime}}\right)\right)^{d_{0}}=-m p_{0}\left(c_{1}\left(\theta_{\chi^{\prime}}\right)\right)^{d_{0}} .
\end{gathered}
$$

Since $\theta_{\chi^{\prime}}$ is ample, we have $\left(c_{1}\left(\theta_{\chi^{\prime}}\right)\right)^{d_{0}}>0$. Therefore,

$$
\left(c_{1}\left(L_{\chi^{\prime}}^{\otimes m q_{0}}\right)\right)^{d_{0}} c_{1}\left(L_{\chi^{\prime}}^{\otimes m q_{0}} \otimes h^{*} \mathcal{O}_{X}\left(-m p_{0} x_{0}\right)\right)<0 .
$$

This means that the restriction of the line bundle $L_{\chi^{\prime}}^{\otimes m q_{0}}$ to the effective divisor $\operatorname{Div}\left(\zeta_{0}\right)$ (constructed in Eq. (2.11)) on $E_{G} / P^{\prime}$ is of negative degree (recall that $\operatorname{Div}\left(\zeta_{0}\right)$ defines $\left.L_{\chi^{\prime}}^{\otimes m q_{0}} \otimes h^{*} \mathcal{O}_{X}\left(-m p_{0} x_{0}\right)\right)$; by degree we mean the top exterior product of the first Chern class. But this contradicts the fact that the line bundle $L_{\chi^{\prime}}^{\otimes m q_{0}}$ is numerically effective.

Therefore, $E_{G}$ is semistable, and the proof of the proposition is complete.

\section{PRINCIPAL BUNDLES OVER PROJECTIVE MANIFOLDS}

Let $M$ be a connected complex projective manifold of complex dimension $d$. Fix a very ample line bundle $\xi \in \operatorname{Pic}(M)$ on $M$. For a coherent sheaf $F$ on $M$, define the degree

$$
\text { degree }(F):=\left(c_{1}(F) \cup c_{1}(\xi)^{d-1}\right) \cap[M] \in \mathbb{Z} .
$$

If $F$ is a holomorphic vector bundle defined over a Zariski open dense subset $U \subset M$ such that the complement $M \backslash U$ is of (complex) codimension at least two, then define

$$
\operatorname{degree}(F):=\operatorname{degree}\left(\iota_{*} F\right),
$$

where $\iota: U \hookrightarrow M$ is the inclusion map; note that $\iota_{*} F$ is a coherent sheaf on $M$.

Let $G$ be a connected reductive linear algebraic group defined over $\mathbb{C}$. Let $Z(G) \subset G$ be the center of $G$. A principal $G$-bundle $E_{G}$ over $M$ is called semistable if for any holomorphic reduction of structure group $\left.E_{P} \subset E_{G}\right|_{U}$ to any parabolic subgroup $P \subset$ $G$ over some Zariski open subset $U \subset M$, with $\operatorname{codim}_{\mathbb{C}}(M \backslash U) \geq 2$, and for any nontrivial antidominant character $\chi$ of $P$ which is trivial of $Z(G)$, the associated line bundle $E_{P}(\chi):=\left(E_{P} \times \mathbb{C}\right) / P$ over $U$ satisfies the condition

$$
\operatorname{degree}\left(E_{P}(\chi)\right) \geq 0
$$

(see [Ra], $\mathrm{AB}]$ ); in the above definition of $E_{P}(\chi)$, the action of $g \in P$ sends any $(z, c) \in$ $E_{P} \times \mathbb{C}$ to $\left(z g, \chi\left(g^{-1}\right) c\right)$. 
Equivalently, $E_{G}$ is semistable if for every reduction $\sigma:\left.M \longrightarrow\left(E_{G} / P\right)\right|_{U}$ to a (proper) maximal parabolic subgroup $P$ of $G$, over any Zariski open subset $U \subset M$ of the above type, one has

$$
\operatorname{degree}\left(\sigma^{*} T_{\text {rel }}\right) \geq 0
$$

where $T_{\text {rel }}$ is the relative tangent bundle of the projection $E_{G} / P \longrightarrow M$ (see [Ra, page 131, Lemma 2.1]).

Note that in the special case where $\operatorname{dim} M=1$ and $G$ is a simple group the above definition of semistability coincides with the one given in Section 2 .

Let $\mathfrak{g}$ be the Lie algebra of $G$. For a holomorphic $G$-bundle $E_{G}$ over $M$, let

$$
\operatorname{ad}\left(E_{G}\right):=\left(E_{G} \times \mathfrak{g}\right) / G
$$

be the adjoint vector bundle over $M$; the action of $g \in G$ sends any $(z, v) \in E_{G} \times \mathfrak{g}$ to $\left(z g, \operatorname{ad}\left(g^{-1}\right)(v)\right)$. It is known that $E_{G}$ is semistable if and only if the vector bundle $\operatorname{ad}\left(E_{G}\right)$ is semistable [AB, page 214, Proposition 2.10].

Let $P \subset G$ a parabolic subgroup and $\chi$ a character of $P$. So we have an associated line bundle

$$
L_{\chi}:=\left(E_{G} \times \mathbb{C}_{\chi}\right) / P
$$

over $E_{G} / P$; the action of $g \in P$ sends any $(z, c) \in E_{G} \times \mathbb{C}$ to $\left(z g, \chi\left(g^{-1}\right) c\right)$. Let

$$
\phi: E_{G} / P \longrightarrow M
$$

be the natural projection. Any fiber of $\phi$ is isomorphic to $G / P$.

Remark 3.1. Let $G$ be a simple linear algebraic group over $\mathbb{C}$. If $\operatorname{dim} M=1$, then a $G-$ bundle $E_{G}$ over $M$ is semistable if and only if the line bundle $L_{\chi}$ in Eq. (3.1) is numerically effective for some parabolic subgroup $P \subsetneq G$ and some nontrivial antidominant character $\chi$ of $P$ (Proposition 2.2). On the other hand, the main theorem of [MR] says that a vector bundle $V$ over $M$ is semistable if and only if the restriction $\left.V\right|_{C}$ is semistable, where $C \subset M$ is a general complete intersection curve of sufficiently large degree hypersurfaces in $M$ (corresponding to $\xi$ ); see [MR, page 221 , Theorem 6.1]. In particular, $\operatorname{ad}\left(E_{G}\right)$ is semistable if and only if $\left.\operatorname{ad}\left(E_{G}\right)\right|_{C}$ is semistable for any such $C$. Therefore, using the criterion (in Proposition 2.2) for semistability of a $G$-bundle over a Riemann surface it follow immediately that $E_{G}$ is semistable if and only if the line bundle $\left.L_{\chi}\right|_{\phi^{-1}(C)}$ is numerically effective, where $L_{\chi}$ (respectively, $\phi$ ) is defined in Eq. (3.1) (respectively, Eq. (3.2)) and $C \subset M$ is a general complete intersection curve of sufficiently large degree hypersurfaces in $M$ (corresponding to $\xi$ ).

Theorem 3.2. Let $E_{G}$ be a holomorphic principal $G$-bundle over a connected projective manifold $M$, where $G$ is a simple linear algebraic group defined over $\mathbb{C}$. Fix a proper parabolic subgroup $P \subset G$ and a nontrivial antidominant character $\chi$ of $P$. The following two statements are equivalent:

(1) The associated line bundle $L_{\chi}:=\left(E_{G} \times \mathbb{C}_{\chi}\right) / P$ over $E_{G} / P$ defined by $\chi$ is numerically effective. 
(2) The $G$-bundle $E_{G}$ is semistable and the second Chern class

$$
c_{2}\left(\operatorname{ad}\left(E_{G}\right)\right) \in H^{4}(M, \mathbb{Q})
$$

vanishes.

Proof. First assume that the line bundle $L_{\chi}$ is numerically effective. Let $C \subset M$ be a connected smooth complex projective curve. Since $L_{\chi}$ is numerically effective, the restriction of $L_{\chi}$ to $\phi^{-1}(C)$ is numerically effective, where $\phi$ is the projection in Eq. (3.2). So using Proposition 2.2 it follows that the restriction $\left.E_{G}\right|_{C}$ of $E_{G}$ to $C$ is semistable.

Since the restriction of $E_{G}$ to every connected smooth complex projective curve in the variety $M$ is semistable, we conclude that the restriction of $\operatorname{ad}\left(E_{G}\right)$ to every connected smooth curve in $M$ is semistable; recall from Remark 3.1 that a $G$-bundle is semistable if and only if its adjoint bundle is semistable (see [AB, page 214, Proposition 2.10] for proof). From this it follows that the vector bundle $\operatorname{ad}\left(E_{G}\right)$ is semistable. Indeed, if $F \subset \operatorname{ad}\left(E_{G}\right)$ is a subsheaf contradicting the semistability condition, then the restriction to $F$ to a smooth curve $C \subset M$ satisfying the two conditions

(1) $C$ is contained in the Zariski open dense subset over which $F$ is a subbundle of $\operatorname{ad}\left(E_{G}\right)$ (the complement of this open set is of codimension at least two),

(2) $C$ is a complete intersection of hypersurfaces in $M$ for the given very ample line bundle $\xi$.

contradicts the semistability condition of $\left.\operatorname{ad}\left(E_{G}\right)\right|_{C}$.

As ad $\left(E_{G}\right)$ is semistable, we conclude that $E_{G}$ is semistable. We still need to show that $c_{2}\left(\operatorname{ad}\left(E_{G}\right)\right)=0$ to be able to conclude that statement (1) implies statement (2).

For a vector bundle $E$ over $M$, let $\mathbb{P}(E)$ denote the projective bundle over $M$ defined by the one-dimensional quotients of the fibers of $E$. The tautological line bundle over $\mathbb{P}(E)$ will be denoted by $\mathcal{O}_{\mathbb{P}(E)}(1)$. A vector bundle $E$ over $M$ is called numerically effective if the line bundle $\mathcal{O}_{\mathbb{P}(E)}(1)$ over $\mathbb{P}(E)$ is numerically effective.

We will use properties of numerically effective vector bundles proved in [DPS]. In [DPS], a holomorphic vector bundle $E$ over a compact complex Hermitian manifold $X$ is called numerically effective if $\mathcal{O}_{\mathbb{P}(E)}(1)$ over $\mathbb{P}(E)$ admits Hermitian connections with arbitrary small negative part of curvature (see [DPS, page 297, Definition 1.2]). For projective manifolds, the above two definitions of numerically effectiveness coincide. Indeed, if $E$ is numerically effective in the sense of [DPS, Definition 1.2], then it is clearly numerically effective when defined using curves in $\mathbb{P}(E)$; see the comment in [DPS, page 297] following Definition 1.2. For the converse direction, take any connected complex projective manifold $X$, and fix an ample line bundle $L$ on $X$. Also, fix a Hermitian connection on $L$ with positive curvature. Let $\Omega_{L}$ be the curvature of this Hermitian connection on $L$, which is positive by assumption. If $\eta$ is a numerically effective line bundle on $X$, then $\eta^{\otimes n} \otimes L$ is ample for each $n \geq 1$ (numerically effectiveness is defined using curves). Take a Hermitian connection on $\eta^{\otimes n} \otimes L$ with positive curvature. This connection on $\eta^{\otimes n} \otimes L$ and the given connection on $L$ together define a Hermitian connection on $\eta$. Indeed, as $\eta^{\otimes n}=$ $\left(\eta^{\otimes n} \otimes L\right) \otimes L^{*}$, there is an induced connection on $\eta^{\otimes n}$, which in turn induces a connection 
on $\eta$. The curvature of this connection on $\eta$ is bounded below by $-\Omega_{L} / n$ (recall that the curvature of the connection on $\eta^{\otimes n} \otimes L$ is positive). Therefore, the line bundle $L$ is numerically effective in the sense of [DPS].

Let

$$
f: C \longrightarrow M
$$

be a holomorphic map from a connected smooth complex projective curve. We will show that the vector bundle $f^{*} \operatorname{ad}\left(E_{G}\right)$ over $C$ is numerically effective. For this we will first prove that the $G$-bundle $f^{*} E_{G}$ is semistable.

There is a natural map

$$
f_{G}:\left(f^{*} E_{G}\right) / P \longrightarrow E_{G} / P
$$

defined using the natural identification $\left(f^{*} E_{G}\right) / P=f^{*}\left(E_{G} / P\right)$. The line bundle $f_{G}^{*} L_{\chi}$ over $\left(f^{*} E_{G}\right) / P$ is identified with the line bundle $L_{\chi}^{\prime}$ associated, for the character $\chi$ of $P$, to the principal $P$-bundle over $\left(f^{*} E_{G}\right) / P$ defined by the projection $f^{*} E_{G} \longrightarrow\left(f^{*} E_{G}\right) / P$. Since $L_{\chi}$ is numerically effective, we conclude that $f_{G}^{*} L_{\chi}=L_{\chi}^{\prime}$ over $\left(f^{*} E_{G}\right) / P$ is also numerically effective [Fu, page 360 , Proposition 2.2]. Consequently, the $G$-bundle $f^{*} E_{G}$ is semistable (Proposition 2.2).

To show that $f^{*} \operatorname{ad}\left(E_{G}\right)$ over $C$ is numerically effective, first note that the vector bundle $f^{*} \operatorname{ad}\left(E_{G}\right)$ is semistable as the $G$-bundle $f^{*} E_{G}$ is semistable. Now, since $\left(f^{*} \operatorname{ad}\left(E_{G}\right)\right)^{*} \cong$ $f^{*} \operatorname{ad}\left(E_{G}\right)$ (any $G$-invariant nondegenerate symmetric bilinear form on $\mathfrak{g}$ gives a nondegenerate symmetric bilinear form on $\left.\operatorname{ad}\left(E_{G}\right)\right)$, we conclude that the line bundle $\bigwedge^{\text {top }} f^{*} \operatorname{ad}\left(E_{G}\right)$ is trivial. This implies that the line bundle

$$
\mathcal{O}_{\mathbb{P}\left(f^{*} \operatorname{ad}\left(E_{G}\right)\right)}\left(\operatorname{dim}_{\mathbb{C}} G\right):=\mathcal{O}_{\mathbb{P}\left(f^{*} \operatorname{ad}\left(E_{G}\right)\right)}(1)^{\otimes\left(\operatorname{dim}_{\mathbb{C}} G\right)}
$$

over the total space of the projective bundle $\mathbb{P}\left(f^{*} \operatorname{ad}\left(E_{G}\right)\right) \longrightarrow C$ is identified with the top exterior product of the relative tangent bundle for the natural projection of $\mathbb{P}\left(f^{*} \operatorname{ad}\left(E_{G}\right)\right)$ to $C$. Using this and the fact that $f^{*} \mathrm{ad}\left(E_{G}\right)$ is semistable it can be deduced from Proposition 2.2 that the line bundle in Eq. (3.4) is numerically effective. To deduce this assertion from Proposition 2.2, consider the principal PGL( $\mathfrak{g})$-bundle $E_{\mathrm{PGL}(\mathfrak{g})}$ over $C$ defined by the projective bundle $\mathbb{P}\left(f^{*} \operatorname{ad}\left(E_{G}\right)\right)$; take the maximal parabolic subgroup $Q^{\prime}$ of PGL $(\mathfrak{g})$ that fixes a hyperplane in $\mathfrak{g}$, and note that the anticanonical line bundle over PGL $(\mathfrak{g}) / Q^{\prime}$ corresponds to an antidominant character of $Q^{\prime}$. As the vector bundle $f^{*} \operatorname{ad}\left(E_{G}\right)$ is semistable, Proposition 2.2 says that the relative anticanonical bundle for the projection $\mathbb{P}\left(f^{*} \operatorname{ad}\left(E_{G}\right)\right) \longrightarrow C$ is numerically effective. Thus the line bundle in Eq. (3.4) is numerically effective.

The line bundle in Eq. (3.4) being numerically effective we conclude that the line bundle $\mathcal{O}_{\mathbb{P}\left(f^{*} \operatorname{ad}\left(E_{G}\right)\right)}(1)$ is numerically effective. In other words, the vector bundle $f^{*} \operatorname{ad}\left(E_{G}\right)$ is numerically effective.

Let

$$
f_{0}: C \longrightarrow \mathbb{P}\left(\operatorname{ad}\left(E_{G}\right)\right)
$$

be a holomorphic map from a connected smooth complex projective curve. Let

$$
p: \mathbb{P}\left(\operatorname{ad}\left(E_{G}\right)\right) \longrightarrow M
$$


be the natural projection. Set $f$ in Eq. (3.3) to be $p \circ f_{0}$. Note that there is a natural map from the projective bundle over $C$

$$
f_{1}: \mathbb{P}\left(f^{*} \operatorname{ad}\left(E_{G}\right)\right)=f^{*} \mathbb{P}\left(\operatorname{ad}\left(E_{G}\right)\right) \longrightarrow \mathbb{P}\left(\operatorname{ad}\left(E_{G}\right)\right)
$$

which projects to $f$. Also, there is a canonical section

$$
\sigma_{1}: C \longrightarrow \mathbb{P}\left(f^{*} \operatorname{ad}\left(E_{G}\right)\right)
$$

(of the projection $\mathbb{P}\left(f^{*} \operatorname{ad}\left(E_{G}\right)\right) \longrightarrow C$ ); the pair $\left(f_{1}, \sigma_{1}\right)$ satisfy the following two conditions

(1) $f_{1} \circ \sigma_{1}=f_{0}$, where $f_{0}$ is defined in Eq. (3.5), and

(2) $f_{1}^{*} \mathcal{O}_{\mathbb{P}\left(\operatorname{ad}\left(E_{G}\right)\right)}(1)=\mathcal{O}_{\mathbb{P}\left(f^{*} \operatorname{ad}\left(E_{G}\right)\right)}(1)$.

We have proved above that $f^{*} \operatorname{ad}\left(E_{G}\right)$ is numerically effective. Using this together with the above two properties of $f_{1}$ and $\sigma_{1}$ we conclude that

$\operatorname{degree}\left(f_{0}^{*} \mathcal{O}_{\mathbb{P}\left(\operatorname{ad}\left(E_{G}\right)\right)}(1)\right)=\operatorname{degree}\left(\left(f_{1} \circ \sigma_{1}\right)^{*} \mathcal{O}_{\mathbb{P}\left(\operatorname{ad}\left(E_{G}\right)\right)}(1)\right)=\sigma_{1}^{*} \operatorname{degree}\left(\mathcal{O}_{\mathbb{P}\left(f^{*} \operatorname{ad}\left(E_{G}\right)\right)}(1)\right) \geq 0$.

Consequently, the vector bundle $\operatorname{ad}\left(E_{G}\right)$ over $M$ is numerically effective.

A vector bundle $E$ is called numerically flat if both $E$ and $E^{*}$ are numerically effective [DPS, page 311, Definition 1.17]. Since $\operatorname{ad}\left(E_{G}\right)^{*} \cong \operatorname{ad}\left(E_{G}\right)$ and $\operatorname{ad}\left(E_{G}\right)$ is numerically effective, we conclude that $\operatorname{ad}\left(E_{G}\right)$ is numerically flat. All the Chern classes of positive degree of a numerically flat vector bundle vanish [DPS, page 311, Corollary 1.19]; in particular, we have $c_{2}\left(\operatorname{ad}\left(E_{G}\right)\right)=0$.

Therefore, statement (1) in the theorem implies statement (2). To prove the converse, assume that $E_{G}$ is semistable and $c_{2}\left(\operatorname{ad}\left(E_{G}\right)\right)=0$.

The semistability of $E_{G}$ implies that the vector bundle $\operatorname{ad}\left(E_{G}\right)$ is semistable $\mathrm{AB}$, Proposition 2.10]. Since $\operatorname{ad}\left(E_{G}\right)^{*} \cong \operatorname{ad}\left(E_{G}\right)$, we have $c_{1}\left(\operatorname{ad}\left(E_{G}\right)\right)=0$, and it is given that $c_{2}\left(\operatorname{ad}\left(E_{G}\right)\right)=0$. Since $\operatorname{ad}\left(E_{G}\right)$ is semistable with $c_{1}\left(\operatorname{ad}\left(E_{G}\right)\right)=0=c_{2}\left(\operatorname{ad}\left(E_{G}\right)\right)$, it admits a filtration of holomorphic subbundles

$$
0=F_{0} \subset F_{1} \subset F_{2} \subset \cdots \subset F_{k-1} \subset F_{k}=\operatorname{ad}\left(E_{G}\right)
$$

such that each $F_{i} / F_{i-1}, i \in[1, k]$, is a stable vector bundle and $c_{j}\left(F_{i} / F_{i-1}\right)=0$ for all $j \geq 1$ and $1 \leq i \leq k$ [Si, page 39, Theorem 2]. To deduce this from [Si, page 39, Theorem 2] simply set the Higgs field in [Si, Theorem 2] to be zero.

Since $F_{i} / F_{i-1}$ is stable and $c_{j}\left(F_{i} / F_{i-1}\right)=0$ for all $j \geq 1$, a theorem due to Donaldson says that each $F_{i} / F_{i-1}$ in Eq. (3.6) admits a unitary flat connection [Do, page 231, Proposition 1].

Let

$$
f: C \longrightarrow E_{G} / P
$$

be a morphism from a connected smooth complex projective curve. We will show that the $G$-bundle $(\phi \circ f)^{*} E_{G}$ over $C$ is semistable, where the map $\phi$ is defined in Eq. (3.2). 
To prove that $(\phi \circ f)^{*} E_{G}$ is semistable, first note that the adjoint bundle $\operatorname{ad}\left((\phi \circ f)^{*} E_{G}\right)$ has a filtration

$$
0=F_{0}^{\prime} \subset F_{1}^{\prime} \subset F_{2}^{\prime} \subset \cdots \subset F_{k-1}^{\prime} \subset F_{k}^{\prime}=(\phi \circ f)^{*} \operatorname{ad}\left(E_{G}\right),
$$

where $F_{i}^{\prime}:=(\phi \circ f)^{*} F_{i}$ with $F_{i}$ is as in Eq. (3.6). It was noted earlier that each $F_{i} / F_{i-1}$ admits a unitary flat connection. A unitary flat connection on $F_{i} / F_{i-1}$ pulls back to induce a unitary flat connection on the pullback $(\phi \circ f)^{*}\left(F_{i}^{\prime} / F_{i-1}^{\prime}\right)=F_{i}^{\prime} / F_{i-1}^{\prime}$. Therefore, $F_{i}^{\prime} / F_{i-1}^{\prime}$, $i \in[1, k]$, is a polystable vector bundle of degree zero [NS, page 560, Theorem 2(A)]. Since $(\phi \circ f)^{*} \operatorname{ad}\left(E_{G}\right)$ is filtered by subbundles with each successive quotient polystable of degree zero, it follows immediately that the vector bundle $(\phi \circ f)^{*} \operatorname{ad}\left(E_{G}\right)$ is semistable. Therefore, the $G$-bundle $(\phi \circ f)^{*} E_{G}$ over $C$ is semistable.

To show that the associated line bundle $L_{\chi}$ over $E_{G} / P$ defined by $\chi$ is numerically effective, take any map $f$ as in Eq. (3.7). Note that $(\phi \circ f)^{*} E_{G}$ (the map $\phi$ is defined in Eq. (3.2)) has a natural reduction of structure group to $P \subset G$ defined by the section

$$
\sigma^{\prime}: C \longrightarrow(\phi \circ f)^{*}\left(E_{G} / P\right) \cong\left((\phi \circ f)^{*} E_{G}\right) / P
$$

that sends any $c \in C$ to $f(c) \in\left(E_{G} / P\right)_{\phi \circ f(c)}=(\phi \circ f)^{*}\left(E_{G} / P\right)_{c}$. There is a natural map

$$
f_{1}:(\phi \circ f)^{*}\left(E_{G} / P\right) \longrightarrow E_{G} / P
$$

such that $f_{1} \circ \sigma^{\prime}=f$ and the line bundle $f_{1}^{*} L_{\chi}$ over $(\phi \circ f)^{*}\left(E_{G} / P\right)$ is identified with $\left((\phi \circ f)^{*} E_{G}\right)(\chi)$, the line bundle over $(\phi \circ f)^{*}\left(E_{G} / P\right)$ associated, for the character $\chi$ of $P$, to the principal $P$-bundle over $\left((\phi \circ f)^{*} E_{G}\right) / P$ defined by the natural projection $(\phi \circ f)^{*} E_{G} \longrightarrow\left((\phi \circ f)^{*} E_{G}\right) / P$.

Now, since $(\phi \circ f)^{*} E_{G}$ is semistable (this was proved earlier), from Proposition 2.2 we conclude that the line bundle $\left((\phi \circ f)^{*} E_{G}\right)(\chi)$ over $(\phi \circ f)^{*}\left(E_{G} / P\right)$ is numerically effective. Consequently, we have

$$
\operatorname{degree}\left(f^{*} L_{\chi}\right)=\left(\sigma^{\prime}\right)^{*} \operatorname{degree}\left(\left((\phi \circ f)^{*} E_{G}\right)(\chi)\right) \geq 0 .
$$

Therefore, $L_{\chi}$ is numerically effective. This completes the proof of the theorem.

Remark 3.3. Let $\kappa \in \mathrm{Sym}^{2} \mathfrak{g}^{*}$ be the Killing form on the Lie algebra $\mathfrak{g}$ of $G$. For a principal $G$-bundle $E_{G}$ over $M$, the form $\kappa$ defines a characteristic class

$$
C_{\kappa}\left(E_{G}\right) \in H^{4}(M, \mathbb{R}) .
$$

This characteristic class can be defined as follows. For any $C^{\infty}$ connection $\nabla$ on $E_{G}$, consider the smooth 4 -form $\kappa\left(\Omega_{\nabla}\right)$ on $M$, where $\Omega_{\nabla}$ is the curvature of $\nabla$. This differential form is closed and the de Rham cohomology class defined by it does not depend on the choice of the connection $\nabla$ on $E_{G}$. This cohomology class defined by the differential form $\kappa\left(\Omega_{\nabla}\right)$ coincides with $C_{\kappa}\left(E_{G}\right)$. The second Chern class $c_{2}\left(\operatorname{ad}\left(E_{G}\right)\right) \in H^{4}(M, \mathbb{Q})$ is a positive multiple of $C_{\kappa}\left(E_{G}\right)$ (the multiplication factor is $2 m(G)$, where $m(G)$ is the dual Coxeter number). Therefore, the condition that $c_{2}\left(\operatorname{ad}\left(E_{G}\right)\right)=0$ is equivalent to the condition that $C_{\kappa}\left(E_{G}\right)=0$ (see statement (2) in Theorem 3.2). 


\section{Generalization for Reductive groups}

The following proposition can be viewed as a reformulation of Theorem 3.2 .

Proposition 4.1. Fix a pair $(P, \chi)$, where $P \subset G$ is a proper parabolic subgroup of the simple group $G$ and

$$
\chi: P \longrightarrow \mathbb{G}_{m}=\mathbb{C}^{*}
$$

a nontrivial antidominant character. A principal $G$-bundle $E_{G}$ over a projective manifold $M$ is semistable with $c_{2}\left(\operatorname{ad}\left(E_{G}\right)\right)=0$ if and only if for every pair of the form $(Y, \psi)$, where $Y$ is a compact connected Riemann surface and

$$
\psi: Y \longrightarrow M
$$

a holomorphic map, and every reduction $E_{P} \subset \psi^{*} E_{G}$ of structure group to $P$ of the principal $G$-bundle $\psi^{*} E_{G}$ over $Y$, the associated line bundle $E_{P}(\chi)=\left(E_{P} \times \mathbb{C}_{\chi}\right) / P$ over $Y$ is of nonnegative degree.

Proof. If $E_{G}$ is semistable with $c_{2}\left(\operatorname{ad}\left(E_{G}\right)\right)=0$, then using a result of Simpson we saw in the proof of Theorem 3.2 that $\operatorname{ad}\left(E_{G}\right)$ admits a filtration of subbundles as in Eq. (3.6) such that each successive quotient admits a unitary flat connection. This filtration induces a filtration of subbundles of the pullback $\psi^{*} \operatorname{ad}\left(E_{G}\right)$ with the property that each successive quotient admits a unitary flat connection (the map $\psi$ is as in the statement of the proposition). Consequently, $\psi^{*} \operatorname{ad}\left(E_{G}\right)$, and hence $\psi^{*} E_{G}$, is semistable. The semistability of $\psi^{*} E_{G}$ immediately implies that the line bundle $E_{P}(\chi)$ in the statement of the proposition is of nonnegative degree.

For the converse direction, assume that $E_{P}(\chi)$ is of nonnegative degree for each reduction of structure group $E_{P} \subset \psi^{*} E_{G}$ and each map $\psi$ of the above type.

Take any holomorphic map

$$
\psi_{1}: Y \longrightarrow E_{G} / P,
$$

where $Y$ is compact connected Riemann surface. Set

$$
\psi=\phi \circ \psi_{1},
$$

where $\phi$ is the projection in Eq. (3.2). The $G$-bundle $\psi^{*} E_{G}$ over $Y$ has a tautological reduction of structure group to $P$

$$
\sigma: Y \longrightarrow\left(\psi^{*} E_{G}\right) / P
$$

that sends any point $y \in Y$ to $\psi_{1}(y) \in\left(E_{G} / P\right)_{\phi \circ \psi_{1}(y)}=\left(\left(\psi^{*} E_{G}\right) / P\right)_{y}$.

Let

$$
E_{P}^{Y} \subset \psi^{*} E_{G}
$$

be the principal $P$-bundle over $Y$ defined by the section $\sigma$ constructed above. The line bundle $\psi_{1}^{*} L_{\chi}$ over $Y$ is identified with $E_{P}^{Y}(\chi)$, the line bundle associated to the principal $P$-bundle $E_{P}^{Y}$ for the character $\chi$. Therefore, the given condition that the degree of $E_{P}^{Y}(\chi)$ is nonnegative implies that degree $\left(\psi_{1}^{*} L_{\chi}\right) \geq 0$. Consequently, $L_{\chi}$ is numerically effective. Finally, Theorem 3.2 says that $E_{G}$ is semistable with $c_{2}\left(\operatorname{ad}\left(E_{G}\right)\right)=0$. This completes the proof of the proposition. 
Let $E$ be a holomorphic vector bundle of rank $r \geq 2$ over $M$. Fix an integer $k \in$ $[1, r-1]$. Proposition 4.1 says that $E$ is semistable with $c_{2}(\operatorname{End}(E))=0$ if and only if for every holomorphic map $\psi: Y \longrightarrow M$, where $Y$ is any compact connected Riemann surface, and for every subbundle $F \subset \psi^{*} E$, of rank $k$ (the fixed integer), the degree of $F^{*} \otimes\left(\psi^{*} E / F\right)$ is nonnegative.

Let $G$ be a complex linear algebraic group such that $G=G_{1} \times G_{2} \times \cdots \times G_{\ell}$, where each $G_{i}$ is simple. A principal $G$-bundle $E_{G}$ over $M$ is semistable if and only if each $G_{i}$-bundle, $i \in[1, \ell]$, obtained by extending the structure group of $E_{G}$ using the projection $G \longrightarrow$ $G_{i}$, is semistable. Any proper parabolic subgroup of $G$ is of the form $Q_{1} \times Q_{2} \times \cdots \times Q_{\ell}$, where $Q_{i}$ is either a parabolic subgroup of $G_{i}$ or $Q_{i}=G_{i}$, with at least one $Q_{j}$ a parabolic subgroup.

Theorem 3.2 and Proposition 4.1 together have the following corollary:

Corollary 4.2. Fix a parabolic subgroup $P=Q_{1} \times Q_{2} \times \cdots \times Q_{\ell} \subset G=G_{1} \times G_{2} \times \cdots \times G_{\ell}$, where $Q_{i}, i \in[1, \ell]$, is a proper parabolic subgroup of the simple linear algebraic group $G_{i}$ defined over $\mathbb{C}$. Fix a character $\chi$ of $P$ whose restriction to each $Q_{i}$ is antidominant and nontrivial. Let $E_{G}$ be a principal $G$-bundle over a connected projective manifold $M$. The following are equivalent:

(1) The $G$-bundle $E_{G}$ is semistable and the second Chern class

$$
c_{2}\left(\operatorname{ad}\left(E_{G}\right)\right) \in H^{4}(M, \mathbb{Q})
$$

vanishes.

(2) The associated line bundle $L_{\chi}:=\left(E_{G} \times \mathbb{C}\right) / P$ over $E_{G} / P$ for the character $\chi$ is numerically effective.

(3) For every pair of the form $(Y, \psi)$, where $Y$ is a compact connected Riemann surface and

$$
\psi: Y \longrightarrow M
$$

a holomorphic map, and every reduction $E_{P} \subset \psi^{*} E_{G}$ of structure group to $P$ of the $G$-bundle $\psi^{*} E_{G}$ over $Y$, the associated line bundle $E_{P}(\chi)=\left(E_{P} \times \mathbb{C}\right) / P$ over $Y$ is of nonnegative degree.

Proof. We have

$$
\operatorname{ad}\left(E_{G}\right)=\bigoplus_{i=1}^{\ell} \operatorname{ad}\left(E_{G_{i}}\right),
$$

where $E_{G_{i}}$ is the principal $G_{i}$-bundle obtained by extending the structure group of $E_{G}$ using the projection $G \longrightarrow G_{i}$. As $c_{1}\left(\operatorname{ad}\left(E_{G_{i}}\right)\right)=0$ for each $i \in[1, \ell]$, we have

$$
c_{2}\left(\operatorname{ad}\left(E_{G}\right)\right)=\sum_{i=1}^{\ell} c_{2}\left(\operatorname{ad}\left(E_{G_{i}}\right)\right) \in H^{4}(M, \mathbb{Q}) .
$$

Also, as we noted earlier, $E_{G}$ is semistable if and only if each $E_{G_{i}}$ is semistable. Therefore, Theorem 3.2 (respectively, Proposition 4.1) says that statement (2) (respectively, statement (3)) implies statement (1). Following the proof of Proposition 4.1 we conclude that (2) and (3) are equivalent. 
Assume that statement (1) is valid.

Since $\operatorname{ad}\left(E_{G_{i}}\right)$ is semistable, and $c_{1}\left(\operatorname{ad}\left(E_{G_{i}}\right)\right) \in H^{2}(M, \mathbb{Q})$ vanishes, the Bogomolov inequality says

$$
0 \leq\left(c_{2}\left(\operatorname{ad}\left(E_{G_{i}}\right)\right) \cup c_{1}(\xi)^{d-2}\right) \cap[M] \in \mathbb{Z},
$$

where $\xi$ is the fixed very ample line bundle over $M$ and $d=\operatorname{dim}_{\mathbb{C}} M[\mathrm{Bo}$. From Eq. (4.1) we have

$$
\left(c_{2}\left(\operatorname{ad}\left(E_{G}\right)\right) \cup c_{1}(\xi)^{d-2}\right) \cap[M]=\sum_{i=1}^{\ell}\left(c_{2}\left(\operatorname{ad}\left(E_{G_{i}}\right)\right) \cup c_{1}(\xi)^{d-2}\right) \cap[M] .
$$

On the other hand, from the given condition that $c_{2}\left(\operatorname{ad}\left(E_{G}\right)\right)=0$ we know that the left-hand side of Eq. (4.3) vanishes. Therefore, from Eq. (4.2) it follows immediately that

$$
\left(c_{2}\left(\operatorname{ad}\left(E_{G_{i}}\right)\right) \cup c_{1}(\xi)^{d-2}\right) \cap[M]=0
$$

for each $i \in[1, \ell]$.

As $\operatorname{ad}\left(E_{G_{i}}\right)$ is semistable with $c_{1}\left(\operatorname{ad}\left(E_{G_{i}}\right)\right)=0$ and satisfies Eq. (4.4), we conclude that the vector bundle $\operatorname{ad}\left(E_{G_{i}}\right)$ admits a filtration of subbundles such that each successive quotient has the property that all the Chern classes of positive degree vanish [Si, page 39, Theorem 2]. This immediately implies that $c_{2}\left(\operatorname{ad}\left(E_{G_{i}}\right)\right)=0$ for each $i \in[1, \ell]$. Consequently, using Theorem 3.2 and Proposition 4.1 we conclude that statement (1) implies both statements (2) and (3) (recall that $E_{G}$ is semistable if and only if each $E_{G_{i}}$ is semistable). This completes the proof of the corollary.

Let $G$ be a connected reductive linear algebraic group defined over $\mathbb{C}$. As before, the center of $G$ will be denoted by $Z(G)$. Therefore, $G / Z(G)$ is a product of simple groups. A parabolic subgroup of $G$ is the inverse image, for the projection of $G$ to $G / Z(G)$, of a parabolic subgroup of $G / Z(G)$. We recall that a parabolic subgroup $P$ of $G$ is said to be without any simple factor if the projection of $P / Z(G)$ to each simple factor of $G / Z(G)$ is a proper parabolic subgroup.

A $G$-bundle $E_{G}$ over $M$ is semistable if and only if the $G / Z(G)$-bundle $E_{G} / Z(G)$ is semistable [Ra, page 146, Proposition 7.1].

Therefore, using Corollary 4.2 we have:

Theorem 4.3. Let $G$ be a connected reductive linear algebraic group over $\mathbb{C}$. Fix a proper parabolic subgroup $P \subset G$ without any simple factor, and also fix a character $\chi$ of $P$ such that

(i) the character $\chi$ is trivial on the center $Z(G) \subset G$, and

(ii) the restriction of $\chi$ to the parabolic subgroup, defined by $P$, of each simple factor of $G / Z(G)$ is nontrivial and antidominant.

Let $E_{G}$ be a principal $G$-bundle over a connected projective manifold $M$. Then the following four statements are equivalent: 
(1) The $G$-bundle $E_{G}$ is semistable and the second Chern class

$$
c_{2}\left(\operatorname{ad}\left(E_{G}\right)\right) \in H^{4}(M, \mathbb{Q})
$$

vanishes.

(2) The associated line bundle $L_{\chi}:=\left(E_{G} \times \mathbb{C}_{\chi}\right) / P$ over $E_{G} / P$ for the character $\chi$ is numerically effective.

(3) For every pair of the form $(Y, \psi)$, where $Y$ is a compact connected Riemann surface and

$$
\psi: Y \longrightarrow M
$$

a holomorphic map, and every reduction $E_{P} \subset \psi^{*} E_{G}$ of structure group to $P$ of the principal $G$-bundle $\psi^{*} E_{G}$ over $Y$, the associated line bundle $E_{P}(\chi)=\left(E_{P} \times\right.$ $\left.\mathbb{C}_{\chi}\right) / P$ over $Y$ is of nonnegative degree.

(4) For any pair $(Y, \psi)$ as in (3), the $G$-bundle $\psi^{*} E_{G}$ over $Y$ is semistable.

Note that statement (3) in Theorem 4.3 implies statement (4). Statement (4) follows from statement (3) by using the criterion for semistability of a $G$-bundle over a Riemann surface given by the equivalence of statements (1) and (3).

Remark 4.4. Theorem 4.3 shows that for a principal $G$-bundle $E_{G}$ over $M$, where $G$ is connected reductive, with $c_{2}\left(\operatorname{ad}\left(E_{G}\right)\right)=0$, the condition that $E_{G}$ is semistable does not depend on the choice of the polarization on $M$ needed for defining degree.

\section{CRITERION FOR PARABOliC BUNDLES}

Let $H$ be a connected linear algebraic group over $\mathbb{C}$. Fix $n$ distinct points

$$
D:=\left\{x_{1}, \cdots, x_{n}\right\} \subset X
$$

of a compact connected Riemann surface $X$. We will refer to the points $\left\{x_{i}\right\}_{i=1}^{n}$ as parabolic points.

Let $E_{H}^{\prime}$ be a connected smooth quasiprojective variety over $\mathbb{C}$ and

$$
f: E_{H}^{\prime} \times H \longrightarrow E_{H}^{\prime}
$$

an algebraic action of $H$ on $E_{H}^{\prime}$.

A parabolic $H$-bundle over $X$ with parabolic structure over $D$ is a pair $\left(E_{H}^{\prime}, f\right)$ as above together with a dominant morphism

$$
\psi: E_{H}^{\prime} \longrightarrow X
$$

satisfying the following conditions:

(1) $\psi \circ f=\psi \circ p_{1}$, where $p_{1}$ is the projection of $E_{H}^{\prime} \times H$ to $E_{H}^{\prime}$, or equivalently, the map $\psi$ is equivariant for the action of $H$;

(2) for each point $x \in X$, the action of $H$ on the fiber $\psi^{-1}(x)_{\text {red }}$ is transitive;

(3) the restriction of $\psi$ to $\psi^{-1}(X \backslash D)$ makes $\psi^{-1}(X \backslash D)$ a principal $H$-bundle over $X \backslash D$, that is, the map $\psi$ is smooth over $\psi^{-1}(X \backslash D)$ and the map to the fiber product

$$
\psi^{-1}(X \backslash D) \times H \longrightarrow \psi^{-1}(X \backslash D) \times_{X \backslash D} \psi^{-1}(X \backslash D)
$$


defined by $(z, g) \longmapsto(z, f(z, g))$ is an isomorphism;

(4) for each point $z \in \psi^{-1}(D)_{\text {red }}$, the isotropy at $z$ for the action of $H$ is a finite subgroup of $H$.

See [BBN] for the details. See [MS] for parabolic vector bundles.

For notational convenience, a parabolic $H$-bundle defined as above will be denoted by $E_{*}$.

Given a parabolic $\mathrm{GL}(n, \mathbb{C})$-bundle $E_{*}$ over $X$, using the standard action of $\mathrm{GL}(n, \mathbb{C})$ on $\mathbb{C}^{n}$ the principal $\mathrm{GL}(n, \mathbb{C})$-bundle over $X \backslash D$ defined by $E_{*}$ gives a vector bundle over $X \backslash D$. This vector bundle has a natural extension to $X$, which is constructed using $E_{*}$, that carries the parabolic structure of the parabolic vector bundle corresponding to $E_{*}$.

Let $Y$ be a compact connected Riemann surface and $\Gamma \subset \operatorname{Aut}(Y)$ a finite subgroup of the group of all holomorphic automorphisms of $Y$. Let $E_{H}$ be a principal $H$-bundle over $Y$. A $\Gamma$-linearization of $E_{H}$ is a lift of the action of $\Gamma$ on $Y$ to the total space of $E_{H}$ that commutes with the action of $H$. So a $\Gamma$-linearization of $E_{H}$ is a left action of $\Gamma$ on $E_{H}$ such that for any $\gamma \in \Gamma$, the automorphism of the variety $E_{H}$ defined by it is an isomorphism of the $H$-bundle $E_{H}$ over the automorphism $\gamma$ of $Y$.

Let $E_{*}$ be a parabolic $H$-bundle over $X$ with $D$ as the parabolic divisor. There is a (ramified) finite Galois covering

$$
\phi: Y \longrightarrow X
$$

and a $\Gamma$-linearized $H$-bundle $E_{H}$ over $Y$, where $\Gamma$ is the Galois group of the covering $\phi$, such that $E_{H}$ corresponds to $E_{*}$ (see $[\mathrm{BBN}]$ ). The covering $\phi$ is ramified over $D$ and for any $x \in D$, the order of ramification is a multiple of the order of the isotropy subgroup for any point in $\psi^{-1}(x)_{\text {red }}$. See [KMM, Ch. 1.1, pages 303-305] for the construction of such a covering.

A reduction of structure group to a closed subgroup $P \subset H$ of a parabolic $H$-bundle $E_{*}$ is a holomorphic section

$$
\sigma: X \longrightarrow E_{H}^{\prime} / P
$$

of the natural projection of $E_{H}^{\prime} / P$ to $X$, where $E_{H}^{\prime}$ is the underlying variety for $E_{*}$. Note that $q^{-1}(\sigma(X))$, where $q: E_{H}^{\prime} \longrightarrow E_{H}^{\prime} / P$ is the natural projection, is a parabolic $P$ bundle. Conversely, if $E_{P}^{\prime} \subset E_{H}^{\prime}$ is a parabolic $P$-bundle with the induced action of $P$, then it defines a section $\sigma$ as above. This section $\sigma$ has the property that $q^{-1}(\sigma(X))$ coincides with $E_{P}^{\prime}$.

If $E_{H}$ is a $\Gamma$-linearized principal $H$-bundle over $Y$ corresponding to the parabolic $H$ bundle $E_{*}$ over $X$, then reductions of $E_{*}$ to $P$ are in bijective correspondence with the $\Gamma$-invariant reductions of $E_{H}$ to $P$ (see $\left.[\mathrm{BBN}]\right)$.

Let $G$ be a connected reductive linear algebraic group over $\mathbb{C}$. Let $E_{*}$ be a parabolic $G$-bundle with $E_{G}^{\prime}$ as the underlying $G$-variety. The parabolic $G$ bundle $E_{*}$ is called semistable if for any reduction of structure group $E_{P}^{\prime} \subset E_{G}^{\prime}$ to any parabolic subgroup $P \subset G$ and for any antidominant character $\chi$ of $P$ trivial on the center of $G$ the parabolic degree of the associated parabolic line bundle $E_{P}^{\prime}(\chi)$ is nonnegative. 
If $E_{G}$ is a $\Gamma$-linearized principal $G$-bundle over $Y$ corresponding to the parabolic $G$ bundle $E_{*}$ over $X$, then $E_{*}$ is semistable if and only if $E_{G}$ is so (see [BBN]).

For a parabolic $G$-bundle $E_{*}$ with $E_{G}^{\prime}$ as the underlying variety, let $N=N\left(E_{*}\right)$ be the least common multiple of the order of the isotropy groups for the action of $G$ on $E_{G}^{\prime}$.

Let $E_{P}^{\prime} \subset E_{G}^{\prime}$ be a reduction of structure group to $P$. If $\chi$ is a character of $P$, then $\chi^{N}$ is trivial on all the isotropy subgroups for the action of $P$ on $E_{P}^{\prime}$ (as $N$ is a multiple of the order of each isotropy subgroup). Therefore, the associated line bundle $E_{P}^{\prime}\left(\chi^{N}\right)$ has trivial parabolic structure.

Let $E_{*}$ be a parabolic $G$-bundle, where $G$ is a simple linear algebraic group, over $X$ with $E_{G}^{\prime}$ as the underlying variety. If $\chi$ is a character of a parabolic subgroup $P \subset G$, then we noted that $\chi^{N}$ is trivial on all the isotropy subgroups for the action of $P$ on $E_{G}^{\prime}$, where, as before, $N=N\left(E_{*}\right)$. Therefore, the quotient

$$
E_{G}^{\prime}\left(\chi^{N}\right):=\left(E_{G}^{\prime} \times \mathbb{C}\right) / P
$$

is a line bundle over $E_{G}^{\prime} / P$, with $P$ acting on $\mathbb{C}$ through $\chi^{N}$.

Theorem 4.3 gives the following criteria for semistability of $E_{*}$.

Proposition 5.1. Fix a pair $(P, \chi)$, where $P \subset G$ is a proper parabolic subgroup of the reductive group and $\chi: P \longrightarrow \mathbb{C}^{*}$ a nontrivial antidominant character. The parabolic $G$-bundle $E_{*}$ is semistable if and only if the associated line bundle $E_{G}^{\prime}\left(\chi^{N}\right)$ over $E_{G}^{\prime} / P$ is numerically effective, where $N$ is the least common multiple of the order of the isotropy subgroups for the action of $G$ on $E_{G}^{\prime}$.

The parabolic $G$-bundle $E_{*}$ is semistable if and only if for every (possibly ramified) covering $\psi: Y \longrightarrow X$, and for every reduction of structure group $E_{P}^{\prime} \subset \psi^{*} E_{G}^{\prime}$ to $P$ of the pulled back parabolic $G$-bundle $\psi^{*} E_{G}^{\prime}=Y \times_{X} E_{G}^{\prime}$, the associated line bundle $E_{P}\left(\chi^{N}\right)$ over $Y$ is of nonnegative degree.

Acknowledgements. The first author thanks the International Centre for Theoretical Physics, Trieste, for hospitality, as well as the International Mathematical Union for a travel support. The second author's research is partly supported by the National Research Project "Geometria delle varietà algebriche". The second author is a member of the research group VBAC (Vector Bundles on Algebraic Curves), which is partially supported by EAGER (EC FP5 Contract no. HPRN-CT-2000-00099) and by EDGE (EC FP5 Contract no. HPRN-CT-2000-00101).

\section{REFERENCES}

[AAB] B. Anchouche, H. Azad and I. Biswas, Harder-Narasimhan reduction for principal bundles over a compact Kähler manifold, Math. Ann. 323 (2002), 693-712.

[AB] B. Anchouche and I. Biswas, Einstein-Hermitian connections on polystable principal bundles over a compact Kähler manifold, Amer. Jour. Math. 123 (2001), 207-228.

[BBN] V. Balaji, I. Biswas and D. S. Nagaraj, Ramified G-bundles as parabolic bundles, Jour. Ramanujan Math. Soc. 18 (2003), 123-138.

[BH] U. Bruzzo and D. Hernandez Ruiperez, Semistability vs. nefness for (Higgs) vector bundles, Diff. Geom. Appl. 24 (2006), 403-416. 
[Bo] F. A. Bogomolov, Holomorphic tensors and vector bundles on projective varieties, Math. USSR Izv. 13 (1979), 499-555.

[DPS] J.-P. Demailly, T. Peternell and M. Schneider, Compact complex manifolds with numerically effective tangent bundles, Jour. Alg. Geom. 3 (1994), 295-345.

[Do] S. K. Donaldson, Infinite determinants, stable bundles and curvature, Duke Math. Jour. 54 (1987), 231-247.

[Fu] T. Fujita, Semipositive line bundles, Jour. Fac. Sci. Univ. Tokyo Sect. IA Math. 30 (1983), 353-378.

[KMM] Y. Kawamata, K. Matsuda and K. Matsuki, Introduction to the minimal model problem, Algebraic geometry, Sendai, 1985, 283-360, Adv. Stud. Pure Math., 10, North-Holland, Amsterdam, 1987.

[MR] V. B. Mehta and A. Ramanathan, Semistable sheaves on projective varieties and their restriction to curves, Math. Ann. 258 (1982), 213-224.

[MS] V. B. Mehta and C. S. Seshadri, Moduli of vector bundles on curves with parabolic structures, Math. Ann. 248 (1980), 205-239.

[Mi] Y. Miyaoka, The Chern classes and Kodaira dimension of a minimal variety, Algebraic geometry, Sendai, 1985, 449-476, Adv. Stud. Pure Math., 10, North-Holland, Amsterdam, 1987.

[NS] M. S. Narasimhan and C. S. Seshadri, Stable and unitary vector bundles on a compact Riemann surface, Ann. of Math. 82 (1965), 540-567.

[PS] A. Pressley and G. Segal, Loop Groups, Oxford Mathematical Monographs, Claredon Press, Oxford, 1986.

[RR] S. Ramanan and A. Ramanathan, Some remarks on the instability flag, Tôhoku Math. Jour. 36 (1984), 269-291.

[Ra] A. Ramanathan, Stable principal bundles on a compact Riemann surface, Math. Ann. 213 (1975), 129-152.

[Si] C. T. Simpson, Higgs bundles and local systems, Inst. Hautes Études Sci. Publ. Math. 75 (1992), $5-95$.

School of Mathematics, Tata Institute of Fundamental Research, Homi Bhabha Road, BOMBAY 400005, INDIA

E-mail address: indranil@math.tifr.res.in

Scuola Internazionale Superiore di Studi Avanzati, Via Beirut 2-4, 34013, Trieste, ITALY

E-mail address: bruzzo@sissa.it 